\title{
Intracellular Elemental Mapping using Simultaneous EELS and EDS: A Combined Approach to Quantifying Na, K and Ca
}

\author{
Alexandra A. Sheader ${ }^{1 *}$, Gema Vizcay-Barrena ${ }^{2}$, Roland A. Fleck ${ }^{2}$, Sarah J. L. Flatters ${ }^{3}$ and Peter D. \\ Nellist ${ }^{1}$ \\ 1. Department of Materials, University of Oxford, UK. \\ 2. Centre For Ultrastructural Imaging, King's College London, UK. \\ 3. Wolfson Centre for Age-Related Diseases, Institute of Psychiatry, Psychology and Neuroscience, \\ King's College London, UK. \\ ${ }^{*}$ Corresponding author: alex.sheader@materials.ox.ac.uk
}

The Scanning Transmission Electron Microscope (STEM) is routinely used in the physical sciences to characterize materials at high spatial resolutions. Advances in quantitative imaging techniques such as high-angle annular dark field (HAADF), combined with careful simulation, now permit atom counting sensitivities at the atomic level [1]. In parallel, developments in analytical STEM grant access to chemical information about a sample. In particular, electron energy loss spectroscopy (EELS) and energy dispersive $\mathrm{x}$-ray spectroscopy (EDS) reveal insights into the composition of a given sample, and can themselves be quantified in terms of atom counts [2].

Sodium, potassium and calcium are all key elements in maintaining cellular function within nerve tissue, and small changes in levels of any can have significant physiological impact [e.g. 3]. However, most approaches for measuring these elements both in- and ex-vivo face technical limitations; many conventional techniques lack either the spatial resolution or the sensitivity required to accurately track small changes in these key cellular elements. Recent developments in genetically-encoded fluorescent $\mathrm{Ca}^{2+}$ markers are a promising avenue for dynamic calcium imaging, but targeting of specific organelles of interest remains challenging [4].

While a number of studies have used either EDS or EELS alone to examine questions posed by life sciences [5,6], few have attempted to fully utilize the analytical capabilities of the STEM. This is a result of challenges related to electron beam-induced damage, sample preparation of tissue sections, and that there has been relatively poor sharing of skill-sets of scientists in the "soft and squishy" and the "hard and dry" scientific worlds [7].

We present our recent work examining the viability of a combined, simultaneously acquired, STEM-EDSEELS approach for investigation of $\mathrm{Ca}, \mathrm{K}$ and $\mathrm{Na}$ levels in sub-cellular structures. We show methods of approaching and overcoming challenges associated with acquiring these measurements, including the large carbon background underneath the $\mathrm{Ca} \mathrm{L}_{2,3}$ edge in EELS, and the substantial overlap of the calcium $K_{\alpha}$ and (significantly more abundant) potassium $K_{\beta}$ peaks in EDS [Figure 1].

We discuss advantages and drawbacks of existing quantification techniques for both spectroscopies and demonstrate a new approach using partial cross-sections for absolute measurement. We have, at the sub cellular level, investigated quantification of $\mathrm{K}, \mathrm{Na}$ and $\mathrm{Ca}$ in units of atoms/ $\mu \mathrm{g}$ dry weight. We also discuss important considerations when preparing a sample for this analysis, such as minimizing sample damage and the diffusion of mobile elements [8]. 
References:

[1] K H W van den Bos et al., Advances in Physics X 3 (2018), p. 1480420.

https://doi.org/10.1080/23746149.2018.1480420 (accessed Feb 5th, 2019)

[2] A M Varambhia et al., Micron 113 (2018), p. 69.

[3] S B Park et al., PLOS ONE 6 (2011), e18469. https://doi.org/10.1371/journal.pone.0018469

(accessed Feb 5th, 2019)

[4] J Suzuki, K Kanemaru and M Iino, Biophysical Journal 111 (2016), p. 1119.

[5] J M Mauritz et al., Biophysical Journal 100 (2011), p. 1438.

[6] R D Leapman et al., Ultramicroscopy 49 (1993), p. 225.

[7] E Hanssen et al., Microscopy and Microanalysis 21(S3) (2015), p. 913.

[8] The authors acknowledge use of the South of England Analytical Electron Microscope

(EP/K040375/1), within the David Cockayne Centre for Electron Microscopy, Department of Materials, University of Oxford, alongside financial support provided by the Henry Royce Institute (Grant ref EP/R010145/1). This research was supported by DTP studentship awarded by the University of Oxford Department of Materials (AAS), and a Physiological Society Research Grant 2015 awarded to SJLF.

(a)

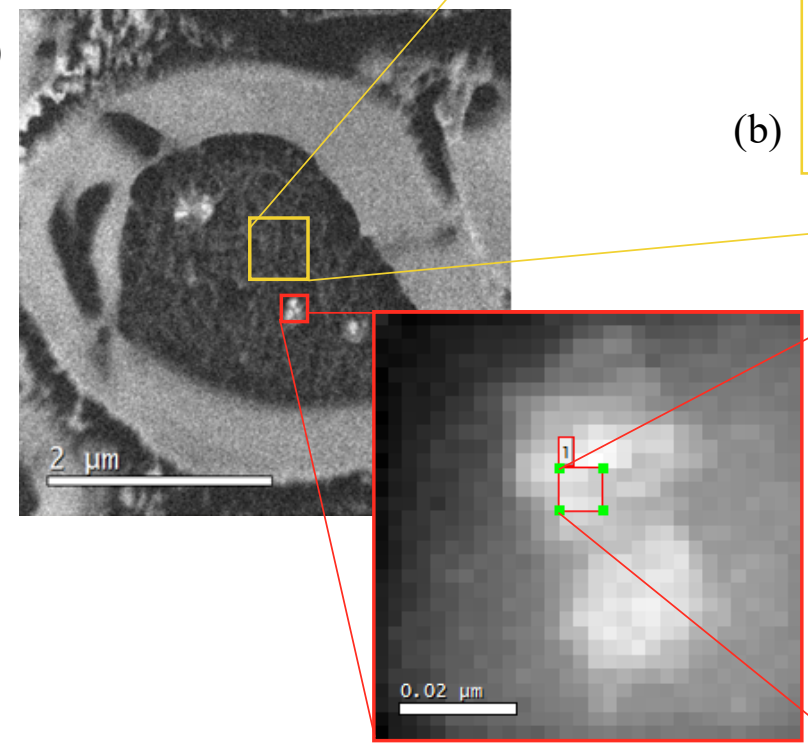

(c) $\mathrm{i}$.

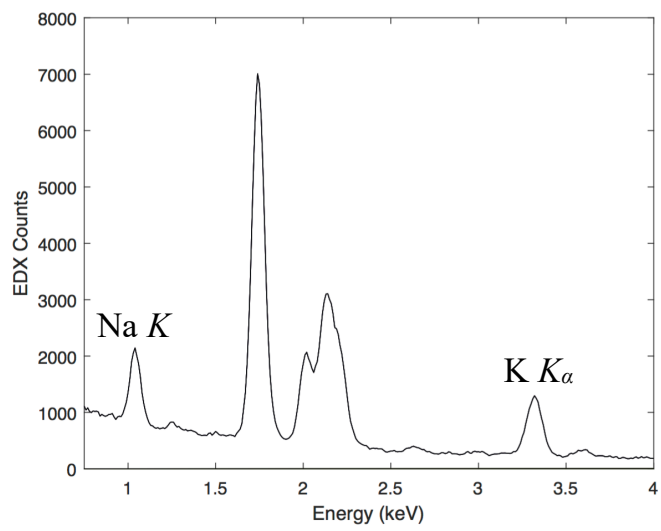

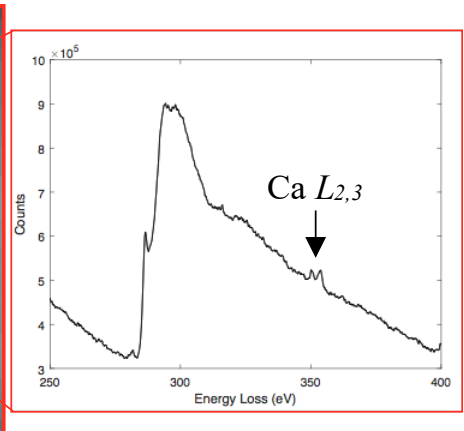

ii.

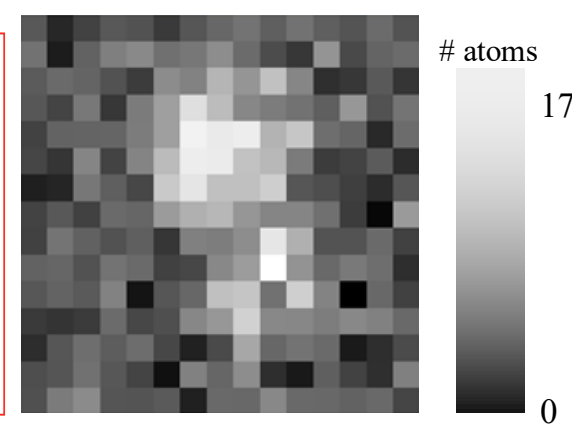

iii.

Figure 1. (a) HAADF image of typical cryosectioned myelinated peripheral nerve cell. (b) EDS obtained from within the lumen of cell shows clear $\mathrm{Na}$ and $\mathrm{K}$ peaks, which are readily quantified. (c) $\mathrm{Ca}$ clusters within a myelinated axon imaged using HAADF (i) can be quantified using EELS (ii). In the resulting chemical map, atom counts range between 0 and 175 atoms per $25 \mathrm{~nm}^{2}$ pixel. 